\title{
Influence of the high voltage pulse shape on the plasma source ion implantation process
}

\author{
R. R. Speth, G. A. Emmert, and M. J. Goeckner ${ }^{a)}$ \\ Engineering Research Center for Plasma-Aided Manufacturing, University of Wisconsin, Madison, \\ Wisconsin 53706-1608
}

(Received 16 June 1994; accepted for publication 26 August 1994)

\begin{abstract}
Using a two fluid model, we investigate the effects on the ion impact energy, of varying the shape of a large, negative, finite rise time, voltage pulse to a planar target. Two pulse shapes are tested, one with exponential shutoff, and one with a powered linear shutoff. For pulse lengths under $50 \mu$ s, we find that pulse shaping can be used to make moderate changes in the energy distribution of the implanted ions. For pulse lengths over $50 \mu \mathrm{s}$, there is a negligible difference in the ion impact energy distribution for the pulse shapes tested. (C) 1994 American Institute of Physics.
\end{abstract}

Ion implantation using plasma source ion implantation (PSII) is a promising ${ }^{1}$ alternative to ion beam implantation. PSII eliminates the need for beam rastering because the target is immersed in the plasma source. PSII also decreases the sputtering of the target because the ions generally impact the target at near-normal incidence. PSII has proven more economical than ion beam implantation in several instances and more applications will undoubtedly be discovered in the near future.

While ion beam implantation has been widely used and studied, PSII is a relatively recent invention. ${ }^{1}$ Because of this, there is a limited knowledge of the basic physics involved in the PSII process. While PSII and ion beam implantation have many similarities, they also have many differences. One important difference is that in a beam system the impacting ions are monoenergetic. This is not the case in PSII systems. Before PSII can be used for industrial applications requiring strict energy tolerances, the spread in the energy of the impacting ions must be reduced.

There are many phenomena that affect the energy distribution of the implanted ions in a PSII system. Some examples are charge exchange collisions, temporal pulse shape, and target charging. ${ }^{2}$ This letter will focus on the effect pulse shaping has on the energy distribution of the impacting ions.

Our study was conducted through the use of a computer simulation based on a two fluid model. The two fluid theory is widely ${ }^{3}$ used to model plasma sheaths. A number of simplifying assumptions are usually made when one applies this theory. First, the ions are assumed to be cold. Second, it is assumed that there is no impediment to the flow of electrons to the electrode surface. Third, it is typically assumed that the sheath region is source-free and collisionless. Thus, ions obey the equation of continuity

$$
\frac{\partial n_{i}}{\partial t}+\frac{\partial}{\partial x} n_{i} v_{i}=0,
$$

and the equation of motion

$$
\frac{\partial^{2} x}{\partial t^{2}}=\frac{\partial v_{i}}{\partial t}=-\frac{e}{M} \frac{\partial}{\partial x} \phi(x, t) .
$$

a) Current address: Princeton Plasma Physics Lab., Princeton University, Princeton, NJ 08543.
Here, $M$ is the ion mass, $n_{i}(x), x$, and $v_{i}(x)$ are the ion density, position, and velocity in the sheath, $n_{0}$ and $v_{0}$ are the plasma density and ion velocity at the sheath-plasma boundary, $-e$ is the electron charge, and $\phi(x)$ is the sheath potential relative to the plasma potential. Fourth, the electrons are assumed to be in thermal equilibrium and thus obey the Boltzmann relation

$$
n_{e}(x, t)=n_{0} \exp \left[e \phi(x, t) / T_{e}\right],
$$

where $n_{e}(x)$ and $T_{e}$ are the electron density and temperature. (In this letter, all temperatures are given in units of energy.) Finally, Poisson's equation is obeyed,

$$
\frac{d^{2} \phi}{d x^{2}}=-\frac{e}{\epsilon_{0}}\left(n_{i}-n_{e}\right),
$$

where $\epsilon_{0}$ is the permittivity constant.

Both Widner et al. ${ }^{4}$ and Emmert and Henry ${ }^{5}$ employed this model to examine the dynamics of pulsed sheaths. In particular, Emmert and Henry used Eq. (2) to advance elemental fluid cells one time step with a leapfrog algorithm. The densities of the cells were then updated using Eq. (1). Finally, the electric potential was determined from a linearized form of Eq. (4) by an iterative process. (Boltzmann's relation was used implicitly in the linearized form of Poisson's equation.)

It is important to note that the model as used here is collisionless and sourceless. By neglecting the effects of these important phenomena we focus exclusively on the effect the pulse shape has on the ion impact energy distribution.

The two pulse shapes that were studied in detail are shown in Fig. 1. These shapes were chosen for their experimental simplicity. The exponentially decaying pulse, which occurs naturally, is the pulse shape used on most PSII systems. The powered shutoff pulse was chosen because it is one of the easiest alterations to make to a typical PSII system. (This alteration would require a second high-voltage switch to shutoff the high voltage pulse.) In both cases, the stage voltage is ramped down in time $t_{d}$ and sustained at the maximum voltage for a period of time $t_{m}$. For our analysis we used a maximum stage voltage of $-10 \mathrm{kV}$ and $t_{d}=1 \mu \mathrm{s}$. This approximates the switching rate found in many PSII systems. ${ }^{6}$ For the exponentially decaying case, the voltage is 


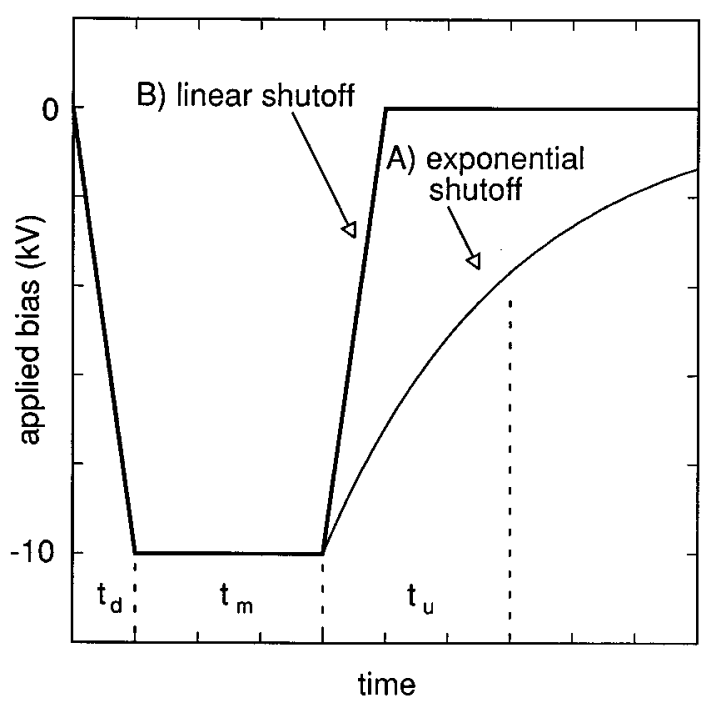

FIG. 1. The temporal pulse shapes examined in this article. The two pulse shapes tested are one with an exponential shutoff (A) and one with a powered, or linear, shutoff (B). These pulse shapes were chosen for their experimental simplicity and suitability. Of the three times shown in the sketch, two were held at fixed values, $t_{d}=1 \mu \mathrm{s}$ and $t_{u}=3 \mu \mathrm{s}$.

allowed to decay up to ground with an exponential time constant of $t_{u}=3 \mu \mathrm{s}$, which simulates the RC time constant occurring naturally in our pulsing network. For the power shutoff case, the voltage is linearly ramped back to zero over a

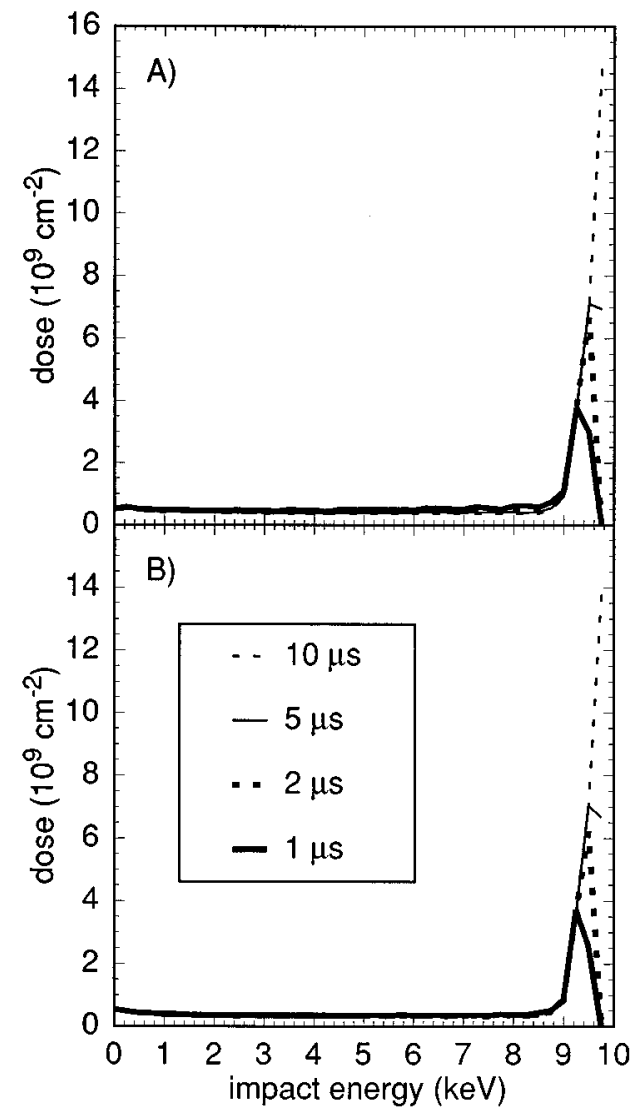

FIG. 2. The energy distribution of the implanted ions as a function of $t_{m}$. (A) The energy distribution for the exponential shutoff pulse. (B) The energy distribution for the powered linear shutoff pulse.

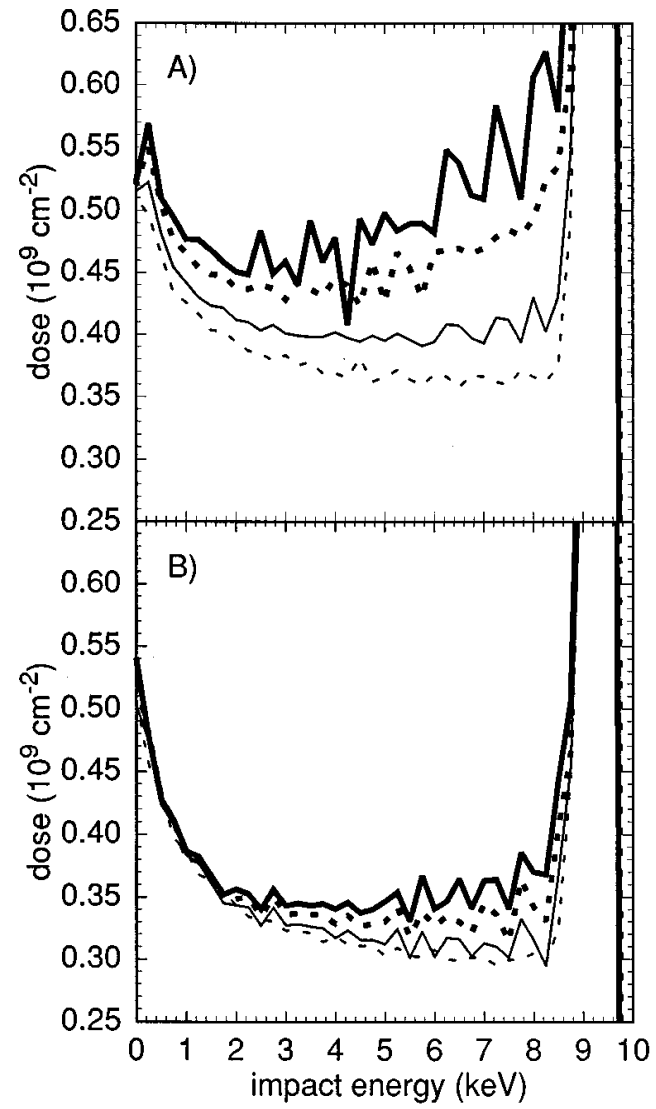

FIG. 3. A closeup of the low energy portion of the ion energy distribution. (A) and (B) are closeups of Figs. 2(A) and 2(B), respectively.

period of $t_{d}$. This is the same pulse shape examined in Ref. 7. Because $t_{m}$ is an adjustable parameter in most systems, $t_{m}$ was varied from 1 to $500 \mu$ s. Finally, we used values for the electron temperature (in units of energy) and density of $1 \mathrm{eV}$ and $10^{9} \mathrm{~cm}^{-3}$, respectively, which are typical for many PSII

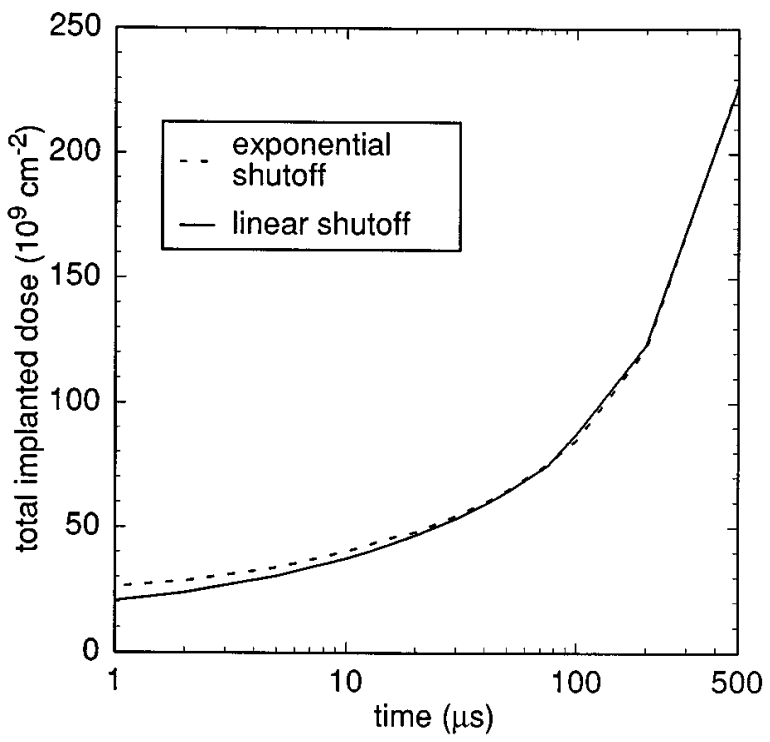

FIG. 4. Total implanted doses as function of $t_{m}$. 
systems. ${ }^{6,8}$ The results obtained for these simulations are shown in Figs. 2-4.

Figures 2 and 3 show the impact energy distributions for various $t_{m}$, where Fig. 3 is a closeup of Fig. 2. Two phenomena are observed in these figures. First, using a linear shutoff reduces the number of low energy ions by almost $30 \%$. This is due to the larger fraction of time the stage is at lower nonzero potential when an exponential decay is used. Second, for longer $t_{m}$ the number of low energy ions is decreased. This is mainly due to the inertia of the ions. In order for an ion to strike the target at the maximum energy, the high voltage pulse must be maintained long enough for that ion to be accelerated to the maximum speed. The number of these low energy ions is determined in part by the speed of the sheath. At very early times in the pulse, several ion plasma periods or less, the sheath expands at a velocity that is many times the ion acoustic velocity. ${ }^{8}$ At later times, the sheath expansion slows to approximately the ion acoustic velocity. Thus, at later times the rate at which ions are engulfed by the sheath is reduced and thus, the number of ions not having sufficient time to reach the maximum velocity is reduced. The speed of the sheath also influences the energy distribution in a second manner. At later times the potential gradient in the sheath is more stable and thus the ions will have more time to respond to the local electric field.

Figure 4 shows the total implanted doses for the two pulse shapes studied. It is seen in Fig. 4, that for small $t_{m}$ the total implanted dose is slightly greater when the pulse is allowed to exponentially decay. This difference in dose arises because the voltage is on longer when the pulse is allowed to exponentially decay. When $t_{m}$ is under $50 \mu$ s this extra on- time is a significant portion of the total pulse, whereas when $t_{m}$ is over $50 \mu$ s the extra on-time becomes negligible when compared to the total pulse length. For $t_{m} \geqslant 50 \mu$ s the total doses from each of the cases are almost identical.

While we have shown that pulse shaping has an effect upon the impacting ion energy distribution, it's influence is probably minor when compared to the affects of collisions, ${ }^{8,9}$ sources, ${ }^{9}$ multidimensional affects, ${ }^{10}$ and target chargeup. ${ }^{2}$ However, while the influence of pulse shape might be small, the use of pulse shaping may permit PSII to be used in applications requiring strict tolerances. ${ }^{11,12}$

${ }^{1}$ J. R. Conrad, J. L. Radke, R. A. Dodd, F. J. Worzala, and N. C. Tran, J. Appl. Phys. 62, 4591 (1987).

${ }^{2}$ G. A. Emmert, J. Vac. Sci. Technol. B 12, 880 (1994).

${ }^{3}$ F. F. Chen, Introduction to Plasma Physics (Plenum, New York, 1974), pp. 244-249.

${ }^{4}$ M. Widner, I. Alexeff, W. D. Jones, and K. E. Lonngren, Phys. Fluids 13, 2532 (1970).

${ }^{5}$ G. A. Emmert and M. A. Henry, J. Appl. Phys. 71, 113 (1992).

${ }^{6}$ M. Shamim, J. T. Scheuer, and J. R. Conrad, J. Appl. Phys. 69, 2904 (1991).

${ }^{7}$ R. A. Stewart and M. A. Lieberman, J. Appl. Phys. 70, 3481 (1991).

${ }^{8}$ M. J. Goeckner, Shamim M. Malik, J. R. Conrad, and R. A. Breun, Phys. Plasmas 1, 1064 (1994).

${ }^{9}$ M. J. Goeckner, R. P. Fetherston, W. N. G. Hitchon, N. C. Horswill, E. R. Keiter, M. M. Shamim, R. A. Breun, J. R. Conrad, and T. E. Sheridan (unpublished).

${ }^{10}$ E. R. Keiter, W. N. G. Hitchon, and M. J. Goeckner, Phys. Plasmas (in press).

${ }^{11}$ L. Zhang, J. H. Booske, R. F. Cooper, J. L. Shohet, J. R. Jacobs, F. S. B. Anderson, M. J. Goeckner, E. B. Wicksberg, and G. Was (unpublished).

${ }^{12}$ L. Zhang, J. L. Shohet, D. Dallmann, J. H. Booske, R. R. Speth, K. Shenai, M. J. Goeckner, J. B. Kruger, P. Rissman, J. E. Turner, E. PerezAlbuerne, S. Lee, and N. Meyyappan, Appl. Phys. Lett. 65, 962 (1994). 